\title{
RESULTS OF TWO BALLOON FLIGHTS FOR THE DETECTION OF HIGH ENERGY $\gamma$-RAYS
}

\author{
M. NIEL, G. VEDRENNE, \\ Centre d'Etude Spatiale des Rayonnements \\ and \\ R. BOUIGUE \\ Observatoire de Toulouse
}

\begin{abstract}
After many years of fruitless research on primary $\gamma$-rays, the results obtained by Clark et al. [1] with the OSO-3 satellite, proved for the first time the existence of a primary $\gamma$-ray flux. The study of the distribution of this radiation showed a strong anisotropy in the direction of the galactic disk and, more precisely, in the direction of the galactic center. Now the production of $\gamma$-rays in the spatial medium is related to high energy processes and to the presence of relativistic electrons. The high energy processes bring about an emission of $\gamma$-rays essentially by decay of the $\pi^{\circ}$ mesons created for instance, by the interaction of cosmic radiation with interstellar matter or by matter-antimatter annihilation. The relativistic electrons can lead to an emission of $\gamma$-rays by various processes: in particular, by bremsstrahlung of the electrons in the interstellar matter or by the Inverse Compton effect with the photons of the stellar light or of the infrared radiation background.
\end{abstract}

These different production processes which have been analyzed in detail [2-3-4-5] enable the estimation of the $\gamma$-rays fluxes at the level of our galaxy and at that of the metagalactic medium. However, because the values obtained by OSO-3 are of an order of magnitude greater than the theoretical estimations, it becomes necessary to explain this disagreement:

either by greatly modifying certain parameters that were used, such as the density of matter. An increase of an order of magnitude in relation to the estimations given by radio observations of the $21 \mathrm{~cm}$ wavelength ray is thus necessary [6].

or, for example, by assuming the contribution of discrete sources [7] without modification of the basic parameters (density, cosmic-ray flux ...)

In the light of these results, it therefore seems very desirable to continue the measure of the energetic $\gamma$-rays because the astrophysic implications linked to their proof are considerable, as much from the cosmological point of view as from that of the study and origin of the primary radiation. This paper gives the results of two flights carried out with a balloon using a detection system for the $\gamma$-rays of energies greater than $100 \mathrm{MeV}$.

\section{Essential Characteristics of the Detection System}

The detector which has already been described [8] is essentially made up of a spark chamber which is set off by a fast coincidence between a Cerenkov detector and a 


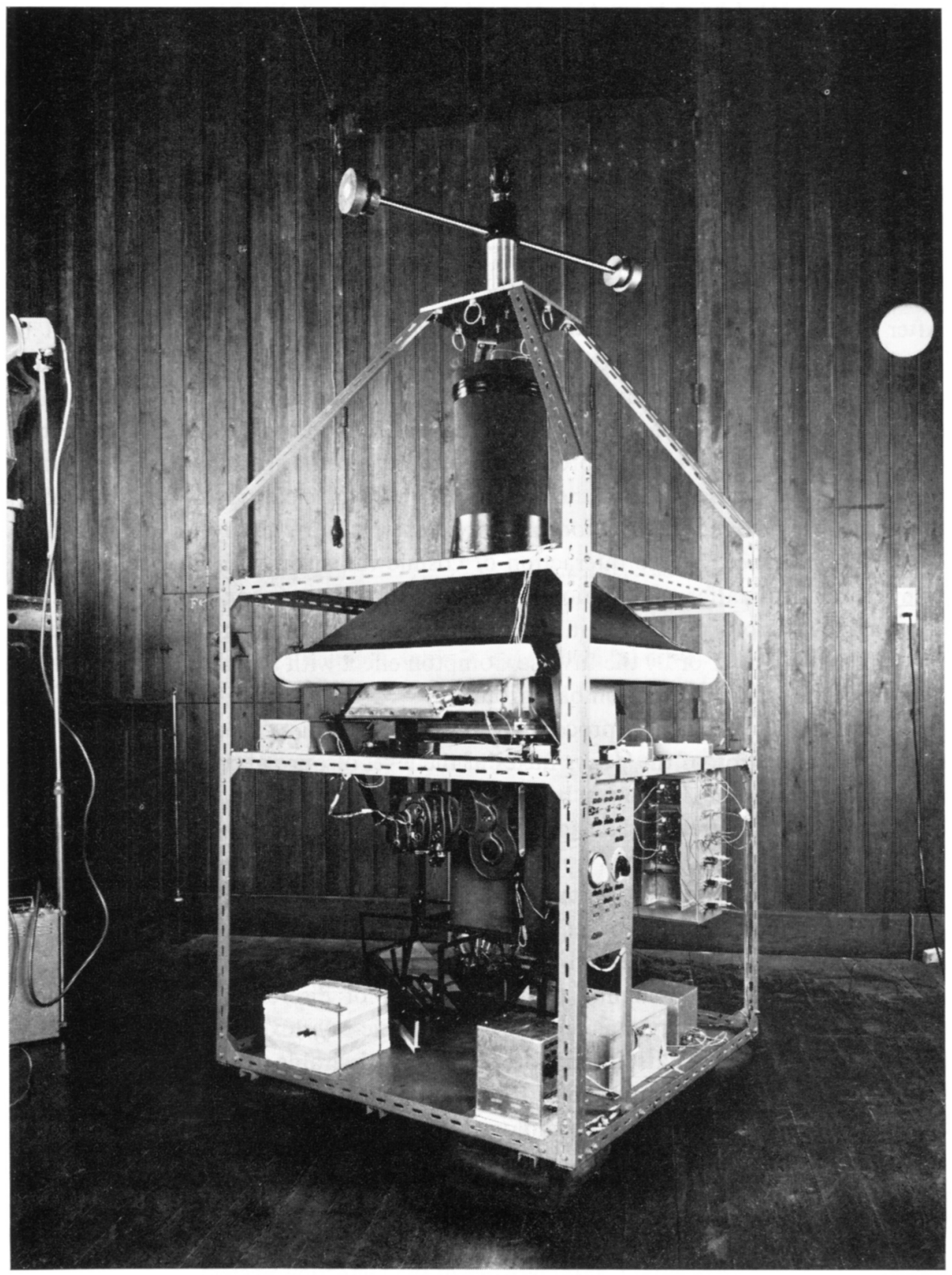

Fig. 1. Detection assembly for the search of $\gamma$-rays with energy above $100 \mathrm{MeV}$. 
plastic scintillation counter. This system is completed by a plastic scintillator which surrounds the spark chamber as much as possible and which enables the elimination of most of the spurious events, and, in particular, those produced by the interaction of particles with the lateral walls of the detector (Figure 1).

In fact, for the two flights whose results are discussed in this paper, the experimental apparatus, although based on the same principle, differs essentially from that in Figure 1 because of the position of the different scintillators. The schematic block diagram of the system used is given in Figure 2. The plastic scintillator, which is in

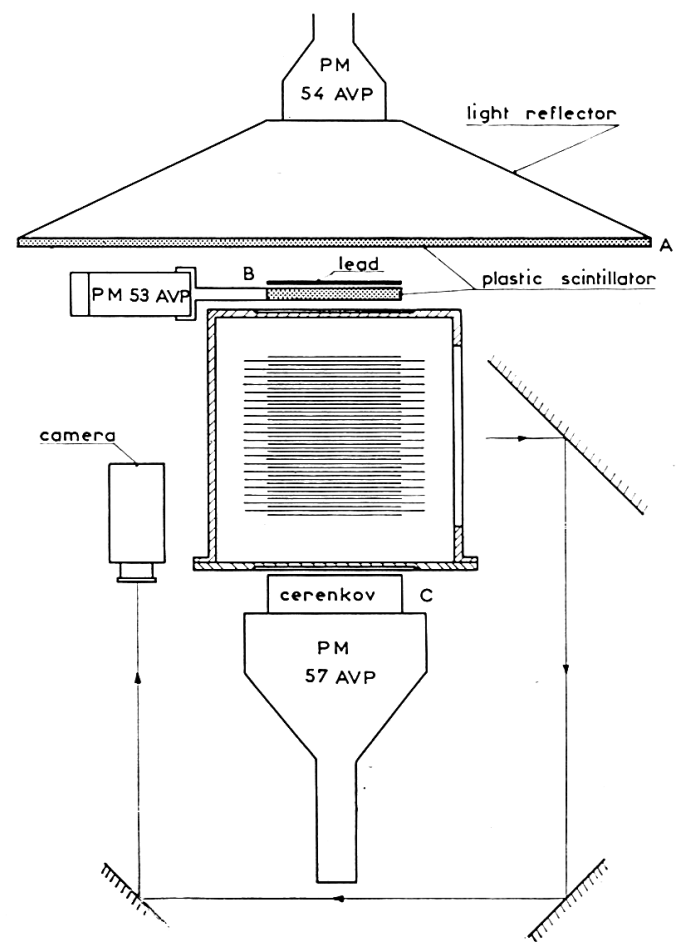

Fig. 2. Synoptic scheme of the detector used for the flights of May and July 1968.

coincidence with the Cerenkov detector, is placed above the chamber $(17 \mathrm{~cm} \times 17 \mathrm{~cm})$ and the conversion of the $\gamma$-rays into $\left(\mathrm{e}^{+}, \mathrm{e}^{-}\right)$pairs takes place in a lead plate. The efficiency of the detector varies from $20 \%$ to $25 \%$ between $100 \mathrm{MeV}$ and $1000 \mathrm{MeV}$. The half angle of aperture of the detection system is $23^{\circ}$ and the geometrical factor is $33 \mathrm{~cm}^{2} \mathrm{sr}$.

\section{Analysis of the Results}

The two flights in May and July 1968, were carried out from AIRE SUR L'ADOUR $\left(43^{\circ} 70 \mathrm{~N}, 0^{\circ} 30 \mathrm{E}\right)$ and GAP $\left(44^{\circ} 5 \mathrm{~N}, 6^{\circ} \mathrm{E}\right)$. The launching times were chosen to explore the same zone of the sky crossing the galactic plane. Presented as an 


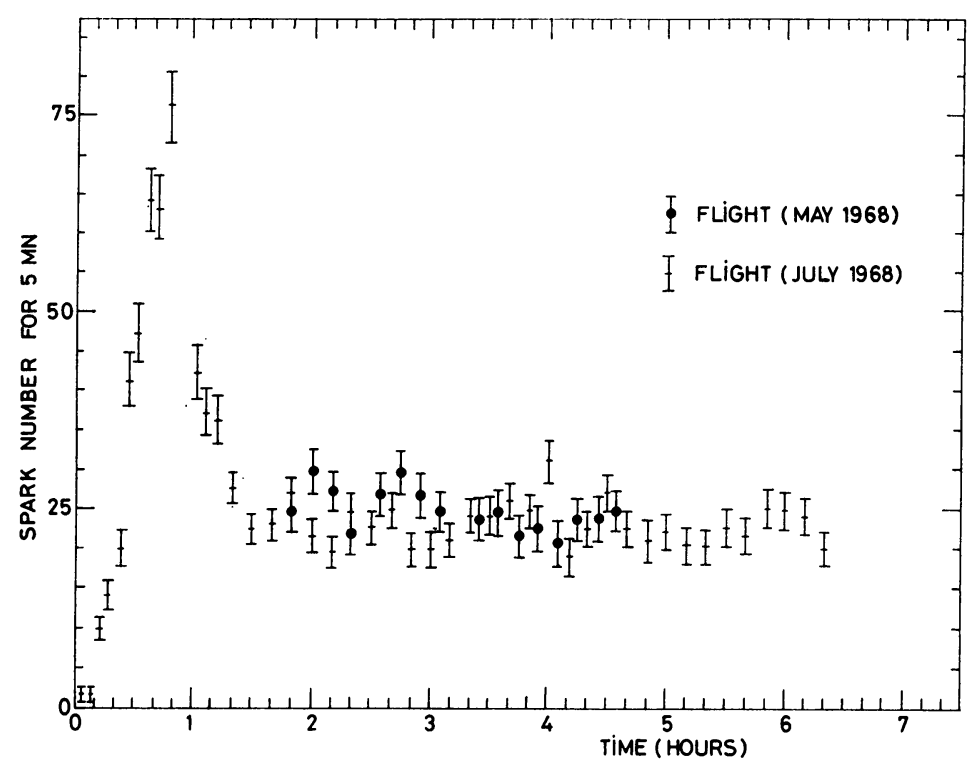

Fig. 3. Variation of the sparks counting rate with time for the 2 flights.

example, Figure 3 shows the comparison of the sparks counting rate during these two flights for a residual pressure of $8 \mathrm{mb}$. The good agreement of the measurements makes it possible to put together the count rates obtained and thus to improve the statistical accuracy of the measured fluxes. In order to interpret these results, however, it is necessary to define the different types of tracks which are to be analyzed and then to reconstitute the arrival direction of the $\gamma$-rays materialized by one or two tracks in the spark chamber. After rejecting the unexploitable negatives, two classes of events were retained, single and double trajectories.

In the first category, the tracks are either straight or curved. The straight tracks can correspond to high energy $\gamma$-rays $\left(\mathrm{e}^{+}\right.$and $\mathrm{e}^{-}$tracks confused), to protons that the anticoincidence system has not eliminated, or to particles associated with the interaction of neutral particles in the chamber or in the walls of the chamber.

The curved trajectories can correspond, for example, to a single prong of an $\left(\mathrm{e}^{+}, \mathrm{e}^{-}\right)$fork if one of the two electrons does not cross the spark chamber or to a Compton interaction in the lead or in the upper part of the chamber.

In the second category, the trajectories are double; that is to say that the $\mathrm{e}^{+}$and $\mathrm{e}^{-}$electron tracks are brought to light, and the presence of an associated $\gamma$-ray is deduced without ambiguity.

This classification leads to the analysis of two parameters:

(a) the arrival direction of the $\gamma$-ray which is either that of the single trajectory or that of the bisector of the tracks corresponding to the $\left(\mathrm{e}^{+}, \mathrm{e}^{-}\right)$pair;

(b) the apparent aperture angle of the fork which allows the measure of the energy of the $\gamma$-ray. 
The analysis of the photographs leads to the classification of the different types of events. A program then gives their characteristics: azimuthal angle, zenithal angle, aperture angle of the forks, restitution of the $y$-rays arrival directions in the $(\alpha, \delta)$ coordinates and then in the $\left(l_{\mathrm{II}}, b_{\mathrm{II}}\right)$ coordinates.

\section{A. ATMOSPHERIC BACKGROUND}

From this analysis and from the variation of the sparks counting rate as a function of time, some verifications that the apparatus was working correctly were undertaken.

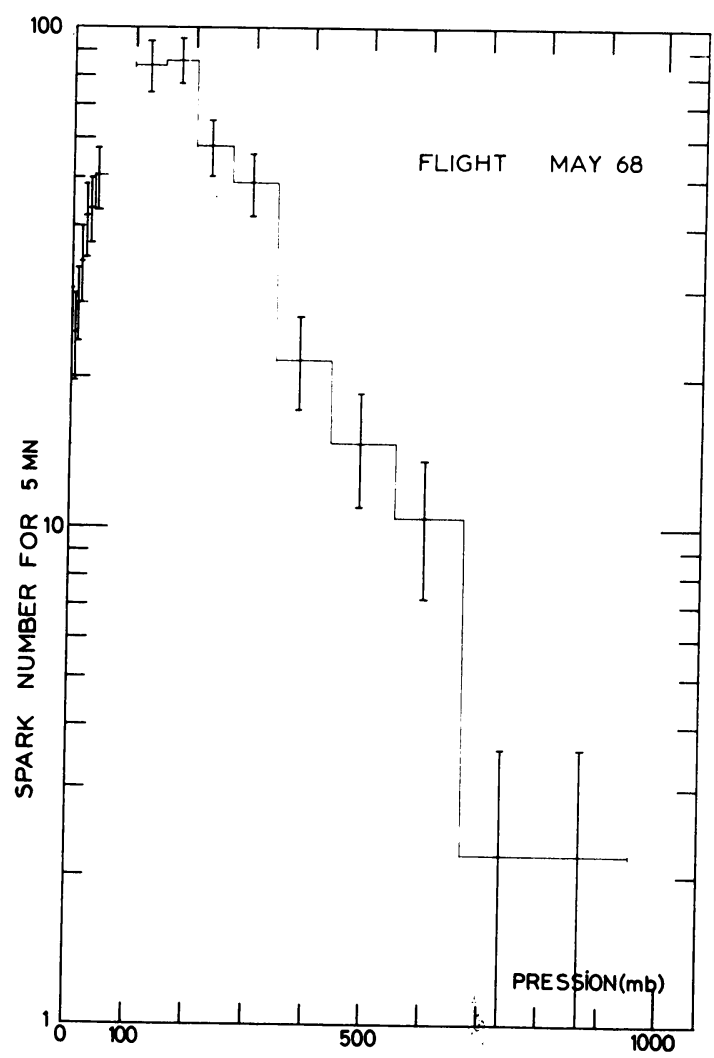

Fig. 4. Variation of $\gamma$-rays flux with residual pressure.

For example, the variation of the sparks counting rate as a function of the pressure between 8 and $1000 \mathrm{mb}$ is in good agreement with the previous experimental results [9], [10], and the curve's maximum is around $180 \mathrm{mb}$ (Figure 4). The detailed study of the part of the curve ranging from 8 to $25 \mathrm{mb}$ cannot, by extrapolation to $0 \mathrm{mb}$, lead to a precise determination of an isotropic primary flux. Moreover, a flux value included between $5.10^{-4}$ and $10^{-3} \mathrm{\gamma} / \mathrm{cm}^{2} \mathrm{~s}$ sr is consistent with the experimental data. 


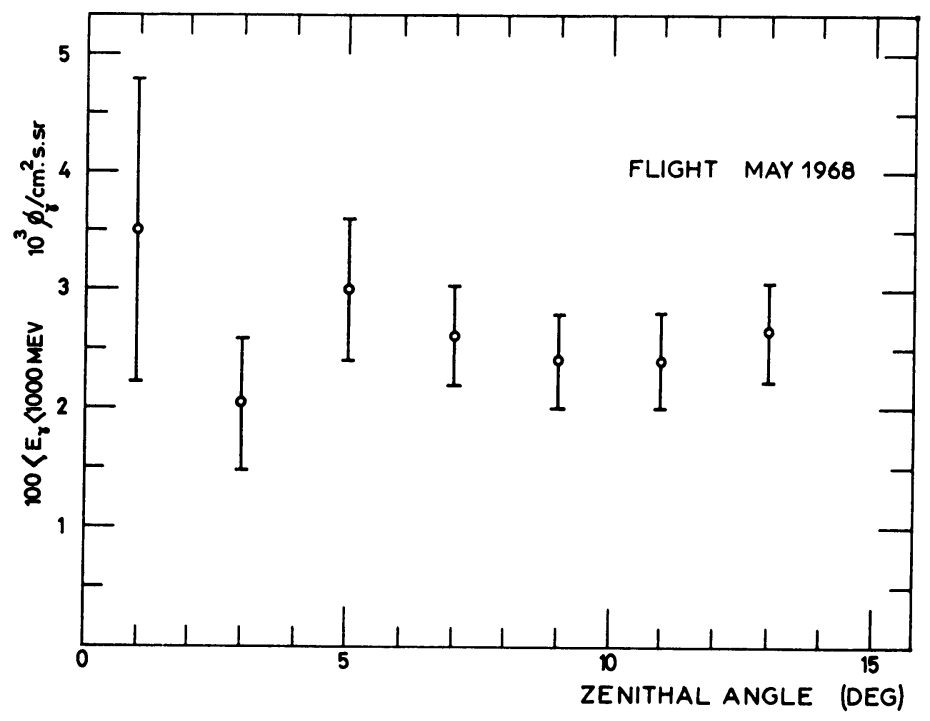

Fig. 5. Variation of $\gamma$-rays flux $\left(100 \mathrm{MeV} \leqslant E_{\gamma} \leqslant 1000 \mathrm{MeV}\right)$ with zenith angle.

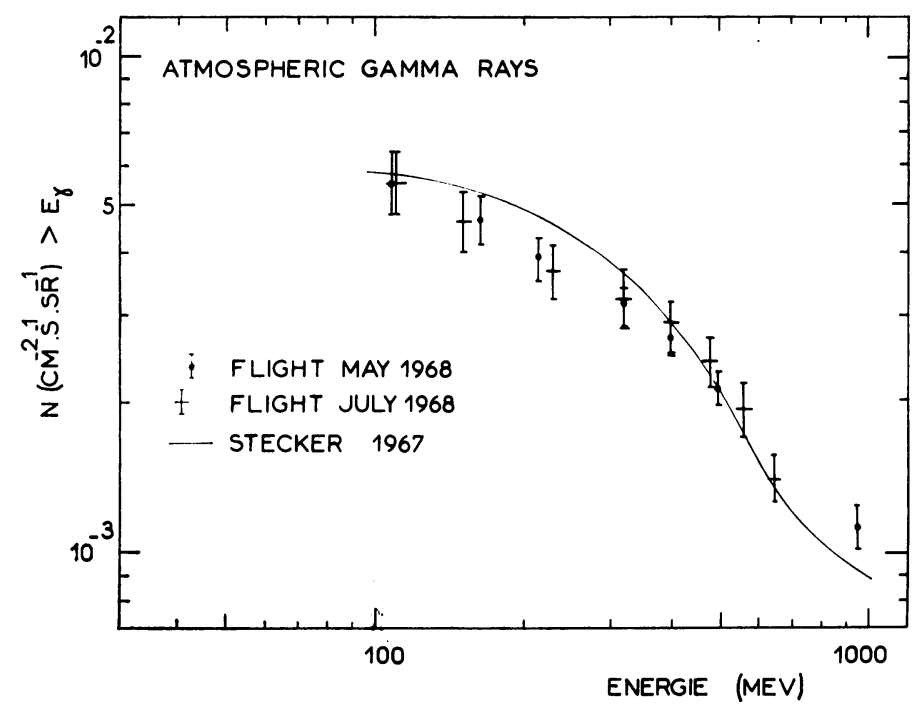

Fig. 6. Integral $\gamma$-rays spectrum observed during the two flights at $8 \mathrm{~g} / \mathrm{cm}^{2}$.

In other respects, for the different types of analyzed events and being given a constant value for the solid angle, the variation of the $\gamma$-ray flux as a function of the zenithal angle $\left(0<\theta<15^{\circ}\right)$ (Figure 5) is isotropic. The anisotropy of this flux of essentially secondary origin comes to light only for greater values of $\theta$ [11], and our results are in good agreement in the common zone $\left(\theta<15^{\circ}\right)$. 
Finally, the energy spectrum of the observed $\gamma$-rays can be deduced from the measurement of the apparent aperture angle of the forks. For this we used the calculations developed by Olsen [12], which take into account the modifications of the aperture angle of the forks by the multiple diffusion process. Figure 6 shows the spectrum obtained; it is in good agreement with the previously published [13], [14], [15] theoretical and experimental results.

\section{B. RESEARCH OF A PRIMARY FLUX}

The sky being divided into elements of constant solid angles, and considering only the forks of angles of less than $6^{\circ}$ the distribution of the $\gamma$-rays detected in $6^{\circ} \times 6^{\circ}$ squares, can be deduced either in the $(\alpha, \delta)$ or in the $\left(l_{\mathrm{II}}, b_{\mathrm{II}}\right)$ coordinates. For each of the sky elements, the exposure time and the secondary origin background have been calculated. The comparison of this background and of the number of $\gamma$-rays actually observed is presented in Figure 7; it does not show any significant flux associated with a definite region. Moreover, the presence of an anisotropy when crossing the galactic plane does not appear in this display. Nevertheless, in order to bring to light an eventual anisotropy, and to make a comparison with the results

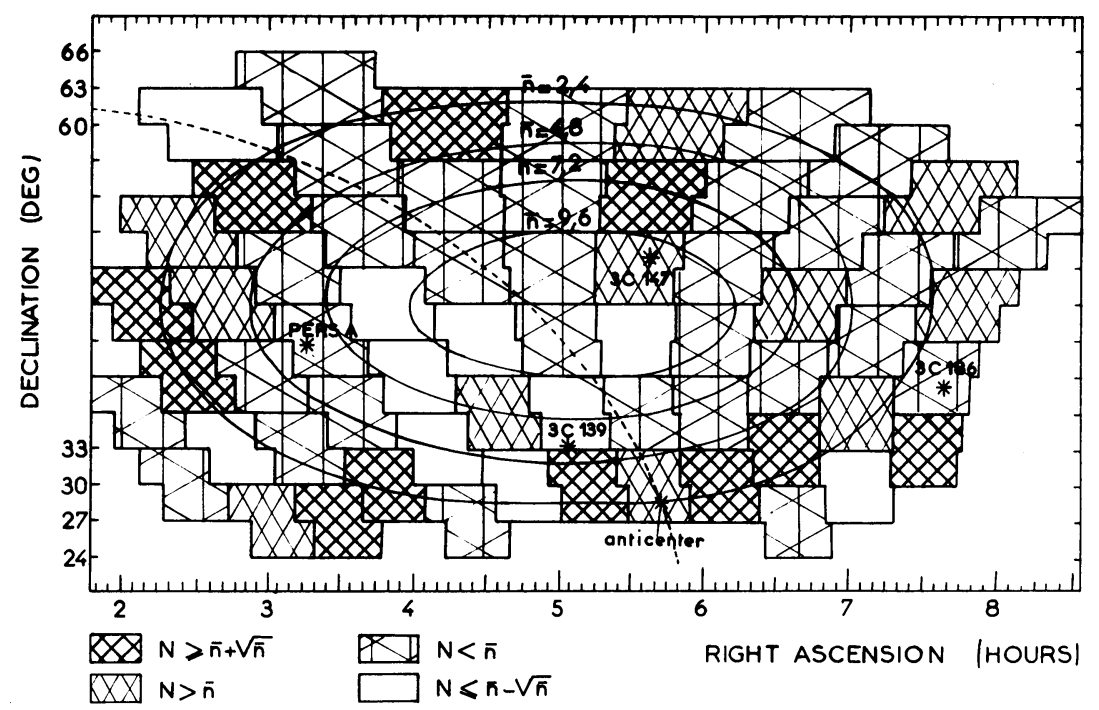

Fig. 7. Angular distribution over the celestial sphere of $\gamma$-rays trajectories. Contour lines represent $\bar{n}$ the average number of events in $6^{\circ} \times 6^{\circ}$ square bins.

already obtained in this area [1], we plotted the distribution of $\gamma$-rays in the $\left(l_{\mathrm{II}}, b_{\mathrm{II}}\right)$ galactic coordinates system. For that the number of sparks observed was counted as a function of $b_{\mathrm{II}}$ for a wide range of galactic longitude $\left(130^{\circ}<l_{\mathrm{II}}<183^{\circ}\right)$ and for the same area-time factor (Figure 8).

No conclusion can be drawn in respect to a flux increase when crossing the galactic 


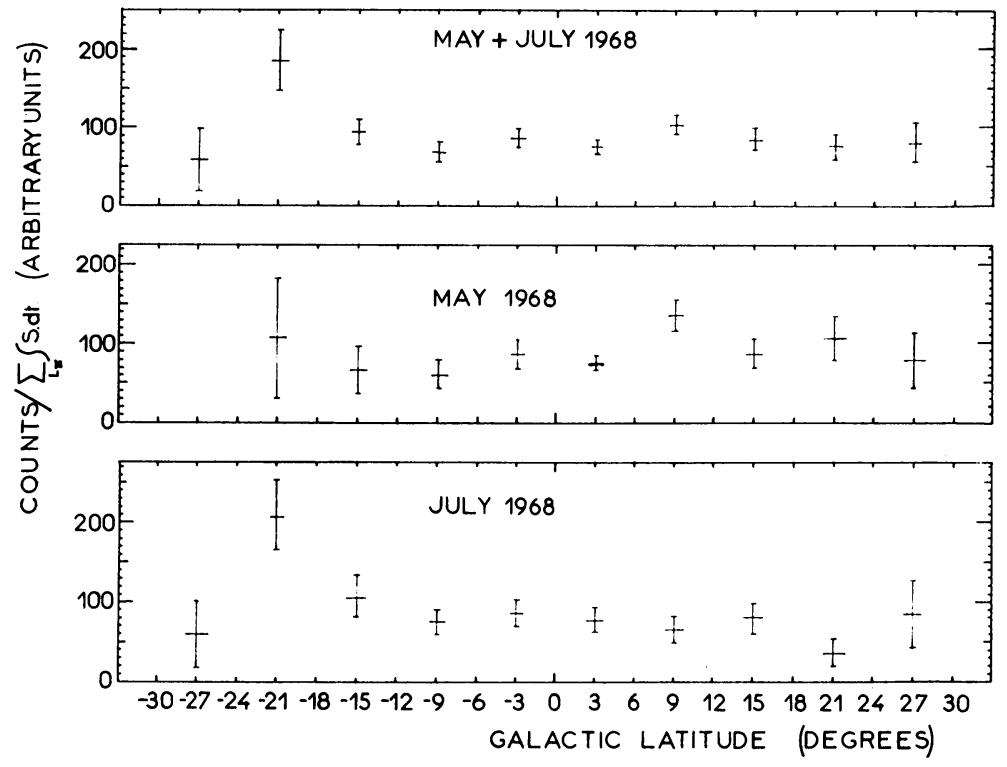

Fig. 8. $\gamma$-Ray intensity plotted against galactic latitude $b_{\text {II }}$.

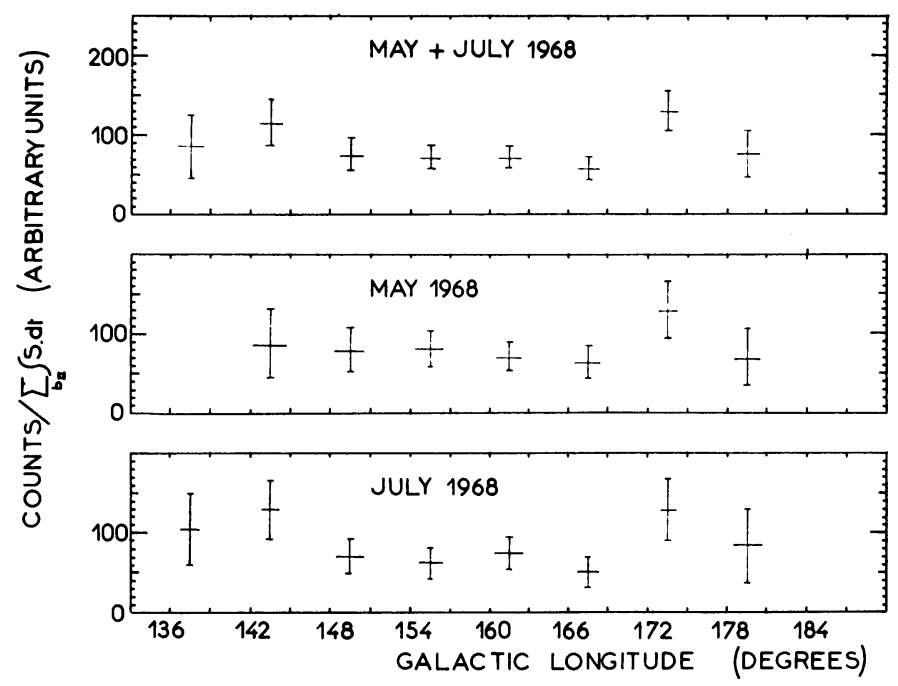

Fig. 9. Variation with galactic longitude $l_{\text {II }}$ of the rate of celestial events for $-9 \leqslant b_{\text {II }} \leqslant+9^{\circ}$.

plane. Nevertheless an anomalously high number of events can be noticed in the vicinity of $b_{\mathrm{II}}=-20^{\circ}$ for the $5 \mathrm{July}$ flight but it is not really statistically significant.

The analogous curve as a function of the galactic longitude does not lead to a sure conclusion (Figure 9). We can notice that the anticenter remained during the flight on the edge of the aperture cone of the detector, and the corresponding exposure time 
was short. Beyond the analysis of the crossing of the galactic plane, the two flights carried out make it possible to give several limit fluxes associated with the punctual sources in transit in the apparatus field during the flight. Unfortunately, the analysis zone which was chosen for the study of the crossing of the galactic plane is not very rich in eventual $\gamma$ sources; thus, only the anticenter and several strong radiosources could be analyzed. The cutting up of the sky dome, as shown in Figure 7 is used to that effect. The average background $\bar{n}$ in one of these elements is thus:

$$
\bar{n}=\Phi_{\mathrm{is}} \cdot \varepsilon \cdot d w \int S d t
$$

$\Phi_{\text {is }}=$ isotropic flux averaged for the whole flight; $\varepsilon=$ detection efficiency; $d w=$ solid angle element; and $\int S d t=$ integral of the surface presented to the source during the flight. This integral is calculated for a direction corresponding to the center of a square bin.

For the May, 1968, flight, a pressure correction had to be added as the balloon descended steadily during the flight. The curves plotted in Figure 7 correspond to a constant background flux.

The limit fluxes associated with the different elements analyzed are calculated from:

$$
\left(m-\frac{\bar{n}}{f}\right) f=\Phi_{\mathrm{Lim}} \cdot \varepsilon \cdot \int S d t
$$

$m$ is the average value such that there exists a $5 \%$ measurement probability of obtaining a count rate less than the count rate $N$ actually observed ( $N=$ total number of forks observed in the bin).

$$
\begin{aligned}
& f=\text { total number of forks/number of forks whose aperture angle is less } \\
& \text { than } 6^{\circ} .
\end{aligned}
$$

The limit fluxes obtained are presented in Table I. This method as noticed by Hearn [16] is not entirely satisfactory; thus, he used the integrated likelihood method as suggested by Greisen. The limit fluxes deduced by this method are presented in Table II and are not very different from the preceding ones, for the actual number of counts observed per square is relatively important.

TABLE I

\begin{tabular}{lll}
\hline Object & $\begin{array}{l}\int S d t \\
\left(\mathrm{~cm}^{2} \mathrm{~s}\right)\end{array}$ & $\begin{array}{l}\text { Limit fluxes } \\
\left(\mathrm{cm}^{2} \mathrm{~s}\right)^{-1}\end{array}$ \\
\hline Pers. A & $3.84 \times 10^{5}$ & $1.4 \times 10^{-5}$ \\
3C 147 & $6.16 \times 10^{5}$ & $3.10^{-5}$ \\
$\alpha=83.3^{\circ}$ & $5.15 \times 10^{5}$ & $1.2 \times 10^{-5}$ \\
$\delta=39.1^{\circ}$ & $2.99 \times 10^{5}$ & $2.9 \times 10^{-5}$ \\
3C 139 & $\begin{array}{l}2.99 \times 10^{5} \\
\text { 3C 186 }\end{array}$ & $5.4 \times 10^{-5}$ \\
Anticenter & $1.79 \times 10^{5}$ & $5.6 \times 10^{-5}$
\end{tabular}

TABLE II

Limit fluxes [16]

$\left(\mathrm{cm}^{2} \mathrm{~s}\right)^{-1}$

$2.3 \times 10^{-5}$

$3.1 \times 10^{-5}$

$1.9 \times 10^{-5}$

$3.4 \times 10^{-5}$

$7.10^{-5}$

$5.9 \times 10^{-5}$ 
Finally, the inaccuracy on the direction of the incident $\gamma$-ray in the case of the forks, taken into account, we have worked out a classification program of the events so that the $\gamma$-rays can be associated with all squares situated inside the aperture angle of the fork it creates. In this way the same $\gamma$-ray is counted in several elements thus assuming an equal emission probability of the $\gamma$-rays in the totality of the aperture angle of the pair. Under these conditions, the distributions obtained in the zones where the background is constant are in all cases comparable to Poisson distributions and thus no anomaly in the fluxes can be considered for the whole of these zones.

In conclusion the analysis of these flights, because of too important a residual atmosphere, makes it impossible to find an anisotropy in crossing the galactic plane. For the same reason, no source could be brought to light and only upper limits of fluxes were associated with several objects. The use of a new detection system (Figure 1) with a greater sensitivity and with a better identification of the $\gamma$-rays ought to lead to positive results for the presence of a primary origin $\gamma$-rays flux.

\section{References}

[1] Clark, G. W., Garmire, G. P., and Kraushaar, W. L.: 1968, Astrophys. J. Letters 153, L 203.

[2] Ginzburg, V. L. and Syrovatsky, S. I.: 1964, Origin of Cosmic Rays.

[3] Garmire, G. and Kraushaar, W. L.: 1965, Space Sci. Rev. 4, 123.

[4] Gould R. J. and Burbidge, G. R.: 1966, Handbuch der Physik 46, No. 2.

[5] Fazio, G. G.: 1967, Ann. Rev. Astron. Astrophys. 5, 481.

[6] Stecker, F. W.: 1969, Nature 222, 865.

[7] Ögelman, H.: 1969, Nature 221, 753.

[8] Niel, M., Cassignol, M., Vedrenne, G., and Bouigue, R.: 1969, Nucl. Instr. Methods. 69, 309.

[9] Cline, T. L.: 1961, Phys. Rev. Letters. 7, 109.

[10] Duthie, J. G., Cobb, R., and Stewart, J.: 1966, Phys. Rev. Letters 17, 263.

[11] Fichtel, C. E., Kniffen, D. A., Ögelman, H. B.: COSPAR 69 (Rome), to be published.

[12] Olsen, H.: 1963, Phys. Rev. 131, 406.

[13] Stecker, F. W.: 1968, Nasa TMX 63 181, April.

[14] Fazio, G. G., Helmken, H. F., Cavrak, S. J., and Hearn, D. R.: 1968, Can. J. Phys. 46, S427.

[15] Fichtel, C. E., Kniffen, D. A., and Ögelman, H. B.: 1968, NASA Report X 611, 69 58, February.

[16] Hearn, D.: 1969, Nucl. Instr. Methods 70, 200. 